\title{
FORD AND DIRICHLET REGIONS FOR DISCRETE GROUPS OF HYPERBOLIC MOTIONS
}

BY

\author{
P. J. NICHOLLS
}

\begin{abstract}
It is shown that for a discrete group of hyperbolic motions of the unit ball of $\mathbf{R}^{n}$, there is a single construction of fundamental regions which gives the Ford and Dirichlet regions as special cases and which also yields fundamental regions based at limit points. It is shown how the region varies continuously with the construction.

The construction is connected with a class of limit points called Garneti points. The size of the set of such points is investigated.
\end{abstract}

1. Introduction. The aim of this paper is to extend, to higher dimensions, some recent results of the author and A. F. Beardon [2] concerning Ford and Dirichlet regions for Fuchsian groups. We will show that for a discrete group of hyperbolic motions of the unit ball in $\mathbf{R}^{n}, n \geqslant 2$, there is a single construction of fundamental regions which gives the Ford and Dirichlet regions as special cases and which also yields fundamental regions based at limit points. We consider also how the region varies continuously with the construction.

The construction is intimately connnected with a class of limit points now known as Garnett points which were introduced a few years ago by Sullivan [6]. We give an example to show the existence of Garnett points for groups in all dimensions and consider the measure of the set of such points.

The geometric methods employed in [2] for the Fuchsian case are not so convenient in higher dimensions and we adopt in this paper an analytic approach instead, using the Poisson kernel function. Even restricted to the Fuchsian case this approach seems preferable in that some of the proofs are more direct and in one case we are able to improve the result (compare Theorem 1 of [2] with Theorem 1 of the present paper).

2. Preliminaries. We shall be concerned almost exclusively with Möbius transforms preserving the unit ball in $\mathbf{R}^{n}, n \geqslant 2$. We denote by $B$ the unit ball and $S$ the unit sphere in $\mathbf{R}^{n}$, thus

$$
B=\{x:|x|<1\} \text { and } S=\{x:|x|=1\} .
$$

The Poincaré metric $\rho$ is derived from the differential $d \rho=2|d x| /\left(1-|x|^{2}\right)$ where the geodesics are the circular arcs orthogonal to $S$.

Received by the editors August 17, 1982 and, in revised form, March 21, 1983.

1980 Mathematics Subject Classification. Primary 20H10; Secondary 30 F35.

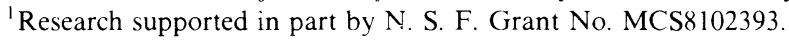

1984 American Mathematical Society $0002-9947 / 84 \$ 1.00+\$ .25$ per page 
Now suppose $\gamma$ is a Möbius transform acting in $\mathbf{R}^{n}$. We denote the Jacobian matrix of $\gamma$ at $x$ by $\gamma^{\prime}(x)$. This matrix is a positive multiple of an orthogonal matrix and this multiple, which measures the change of linear scale, will be denoted by $\left|\gamma^{\prime}(x)\right|$. It is known [1, p. 165] that, for any $x, y \in \mathbf{R}^{n}$,

$$
|\gamma(x)-\gamma(y)|=\left|\gamma^{\prime}(x)\right|^{1 / 2}\left|\gamma^{\prime}(y)\right|^{1 / 2}|x-y| \text {. }
$$

If $\gamma$ preserves $B$ then for any $x \in B$

$$
1-|\gamma(x)|^{2}=\left|\gamma^{\prime}(x)\right|\left(1-|x|^{2}\right)
$$

see, for example, [1, p. 166]. From (1) and (2) we observe that if $z \in B, w \in \bar{B}$, $z \neq w$ and $\gamma$ is Möbius preserving $B$, then

(3) $\left(1-|z|^{2}\right)\left(1-|w|^{2}\right)|z-w|^{-2}=\left(1-|\gamma(z)|^{2}\right)\left(1-|\gamma(w)|^{2}\right)|\gamma(z)-\gamma(w)|^{-2}$.

For $z \in B, w \in \bar{B} \backslash\{z\}$ we define the function $P(z, w)=\left(1-|z|^{2}\right)|z-w|^{-2}$ which is the familiar Poisson kernel (when $n=2$ ). The following lemmas are quite routine but help to fix geometric ideas.

Lemma 1. (i) Suppose $k>0$ and $w \in B$ are fixed. Then the set $\{z: P(z, w)=k\}$ is a noneuclidean sphere centered at $w$ and of radius $\rho$ where

$$
\rho=\log \left\{1+\left(1+k\left(1-|w|^{2}\right)\right)^{-1 / 2}\right\}\left\{1-\left(1+k\left(1-|w|^{2}\right)\right)^{-1 / 2}\right\}^{-1} .
$$

(ii) Suppose $k>0$ and $w \in S$ are fixed. Then the set $\{z: P(z, w)=k\}$ is a sphere internally tangent to $S$ at $w$ and of euclidean radius $(1+k)^{-1}$.

Proof. Suppose $k$ is positive and $w \in B$. Let $\gamma$ be a Möbius transform preserving $B$ and such that $\gamma(w)=0$. We note from (3) that

$$
\{z: P(z, w)=k\}=\left\{z:\left(1-|\gamma(z)|^{2}\right)|\gamma(z)|^{-2}=k\left(1-|w|^{2}\right)\right\}
$$

and this set is equal to

$$
\left\{z:|\gamma(z)|^{2}=\left(1+k\left(1-|w|^{2}\right)\right)^{-1}\right\}
$$

Thus the set of points $\gamma(z)$ forms a circle with center (euclidean and noneuclidean) at $0(=\gamma(w))$ and of noneuclidean radius $\rho$ where $\rho$ is as given in the lemma.

To prove part (ii) suppose $k$ is positive and $w \in S$. If $z \in B$ with $P(z, w)=k$ then $1-|z|^{2}=k|z-w|^{2}$. Using the scalar product in $\mathbf{R}^{n}$ we see then that

$$
1-|z|^{2}=k(z-w) \cdot(z-w)=k|z|^{2}+k-2 k z \cdot w
$$

and so $z \cdot w=\left\{k-1+(k+1)|z|^{2}\right\} / 2 k$. Using this we observe that

$$
\begin{aligned}
|k(z-w)+z|^{2} & =(k(z-w)+z)(k(z-w)+z) \\
& =k^{2}|z-w|^{2}+|z|^{2}+2 k|z|^{2}-\left\{k-1+(k+1)|z|^{2}\right\}=1
\end{aligned}
$$

It follows that if $P(z, w)=k$, then $\left|z-k(1+k)^{-1} w\right|=(1+k)^{-1}$ and the result follows. 
If $\gamma$ is a Möbius transform preserving $B$ and $w \in B \cup S$ then we define

$$
L(\gamma w)=\{z \in B: P(z, w)=P(\gamma(z), w)\}
$$

and we have the following result.

LeMma 2. Suppose $\gamma$ is a Möbius transform preserving $B$.

(i) If $w \in B$ then

$$
L(\gamma, w)=\{z \in B: \rho(z, w)=\rho(\gamma(z), w)\}=\left\{z \in B: \rho(z, w)=\rho\left(z, \gamma^{-1}(w)\right)\right\} \text {. }
$$

(ii) If $w \in S$ and $\gamma$ does not fix $w$ then $L(\gamma, w)$ is the part in $B$ of a sphere orthogonal to $S$. In fact, if $U$ is a Möbius transform which maps $B$ to the upper half-space of $\mathbf{R}^{n}$ with $U(w)=\infty$, then $U(L(\gamma, w))$ is the isometric sphere of the transform $U \gamma U^{-1}$.

Proof. To prove part (i) we consider a Möbius transform $V$ which preserves $B$ and maps $w$ to the origin. Using equation (3) we see that

$$
P(z, w)=\left(1-|V(z)|^{2}\right)\left(1-|w|^{2}\right)^{-1}|V(z)|^{-2}
$$

and that

$$
P(\gamma(z), w)=\left(1-|V \gamma(z)|^{2}\right)\left(1-|w|^{2}\right)^{-1}|V \gamma(z)|^{-2}
$$

Thus

$$
\begin{aligned}
L(\gamma, w) & =\{z \in B:|V(z)|=|V \gamma(z)|\}=\{z \in B: \rho(V(z), 0)=\rho(V \gamma(z), 0)\} \\
& =\{z \in B: \rho(z, w)=\rho(\gamma(z), w)\}
\end{aligned}
$$

by the invariance of the metric $\rho$, and this is the desired result.

We now prove part (ii) and thus suppose that $U$ is a Möbius transform as given in the lemma. We note that $w=U^{-1}(\infty)$ and choose $x \neq w$ in $S$ setting $y=U(x)$. If $z \in L(\gamma, w)$ then, by continuity of $P(z, w)$, we see that

$$
\left(1-|z|^{2}\right)|z-x|^{-2}\left\{\left(1-|\gamma(z)|^{2}\right)|\gamma(z)-x|^{-2}\right\}^{-1} \rightarrow 1 \quad \text { as } x \rightarrow w .
$$

From (2) we see that the expression above equals

$$
\left|\gamma^{\prime}(z)\right|^{-1}\left|U^{-1} U \gamma(z)-U^{-1}(y)\right|^{2}\left|U^{-1} U(z)-U^{-1}(y)\right|^{-2}
$$

which, from (1), equals

$$
\left|U^{\prime}(x)\right|\left|\gamma^{\prime}(z)\right|^{-1}\left|U^{\prime}(\gamma(z))\right|^{-1}|U \gamma(z)-y|^{2}|U(z)-y|^{-2} \text {. }
$$

Thus, since $x$ converges to $w$ iff $y$ converges to $\infty$, we see that

$$
\left|U^{\prime}(z)\right|\left|\gamma^{\prime}(z)\right|^{-1}\left|U^{\prime}(\gamma(z))\right|^{-1}=1
$$

and so $\left|\left(U \gamma U^{-1}\right)^{\prime}(U(z))\right|=1$. This means that $U(z)$ belongs to the isometric sphere of $U \gamma U^{-1}$ as required.

REMARK. With $\gamma$ as given in the lemma, we observe that the set $\{z \in B$ : $P(z, w)>P(\gamma(z), w)\}$ is a noneuclidean half-space, provided $\gamma(w) \neq w$. If $w \in B$, it is the half-space $\{z \in B: \rho(z, w)<\rho(\gamma(z), w)\}$. If $w \in S$ and $U$ is as given in part (ii) of the lemma then the set above is the $U^{-1}$ image of the exterior of the isometric sphere of $U \gamma U^{-1}$. 
3. The fundamental region. We denote by $\Gamma$ a discrete group of Möbius transforms acting in the unit ball $B$ of $\mathbf{R}^{n}$. If $w \in B \cup S$ is selected then we define the set

$$
D_{w}=\{z \in B: P(z, w)>P(\gamma(z), w) \text { all } \gamma \in \Gamma \backslash I\} .
$$

Note from (3) that $D_{w}$ is empty if any element of $\Gamma$ fixes $w$ and, from the remark following Lemma $2, D_{w}$ is a countable intersection of half-spaces and is thus convex in the noneuclidean sense. Clearly $D_{w}$ can never contain two $\Gamma$-equivalent points and, in fact, it follows from Lemma 2 that if $w \in B$ and is not fixed by any element of $\Gamma$ (except the identity), then $D_{w}$ is the Dirichlet region centered at $w$. If $w \in S$ is not a limit point of $\Gamma$ and is not fixed by any element of $\Gamma$ (except the identity) then $D_{w}$, is the image of the Ford region for a conjugate group.

Even when $w$ is a limit point for the group there is good reason to suspect that $D_{w}$ may well be a fundamental region. In later sections we explain these ideas more fully and will classify those limit points $w$ for which $D_{w}$ is a fundamental region. In the next section discuss how $D_{w}$ varies with $w$.

4. Continuity. We recall the definition of convergence of a sequence of sets $\left\{A_{i}\right\}$ in $\mathbf{R}^{n}$. Defining $\lim \inf A_{i}=\cup_{i=1}^{\infty} \bigcap_{k \geqslant i} A_{k}$ and $\lim \sup A_{i}=\bigcap_{i=1}^{\infty} \cup_{k \geqslant i} A_{k}$, we say that $\left\{A_{i}\right\}$ converges to $A$ if $\lim \inf A_{i}=\lim \sup A_{i}=A$. Given a sequence $w_{n}$ in $B$ which converges to $w$ in $\bar{B}$ we ask whether the regions $D_{w_{n}}$ converge to $D_{n}$. If $w \in B$ this is almost trivial to prove. In the case where $w \in S$ we have the following result. This result, in the case $n=2$, improves on a result previously obtained by the author and A. F. Beardon [2, Theorem 1].

THEOREM 1. Let $\Gamma$ be a discrete group acting in $B$ and $w \in S$. If $w$ is not fixed by any element of $\Gamma$ (except the identity) and if $w_{n}$ is a sequence in $B$ converging to $w$ then $\lim \sup D_{w_{n}} \subset \overline{D_{u}}$. If we further assume that $w_{n}$ converges to $w$ in a cusp then $D_{w} \subset \lim \inf D_{w_{n}}$.

Proof. Choose $\xi \in B$ and define the sets $A_{n}(\xi)=\left\{z \in B: P\left(z, w_{n}\right)>P\left(\xi, w_{n}\right)\right\}$. By continuity of the function $P$ in the second variable we see that

$$
\{z: P(z, w)>P(\xi, w)\} \subset \liminf A_{n}(\xi)
$$

and

$$
\limsup A_{n}(\xi) \subset\{z: P(z, w) \geqslant P(\xi, w)\} .
$$

Now if $\xi \notin \bar{D}_{n}$ then, by the remark following Lemma 2, we see that $P(\gamma(\xi), w)>$ $P(\xi, w)$ for some $\gamma \in \Gamma$. Thus, from (4), $\gamma(\xi) \in \liminf A_{n}(\xi)$ and this means that for $n$ large enough $\xi \notin D_{w_{n}}$ and so $\xi \notin \lim \sup D_{w_{n}}$. This proves the first part of the theorem.

To prove the second part of the theorem we choose $\xi \in B$ and suppose that $\xi \in D_{w}$ and $\xi \notin \lim \inf D_{w_{n}}$, where $w_{n}$ converges to $w$ in a cusp. For infinitely many $n, \xi \notin D_{w_{n}}$ and there exists a sequence $\gamma_{n}$ of elements of $\Gamma$ with $\gamma_{n}(\xi) \in \overline{A_{n}(\xi)}$. However, the set $\{z: P(z, w)<P(\xi, w)\} \cap\left(\cup_{n=1}^{\infty} \overline{A_{n}(\xi)}\right)$ is compact in $B$ (we prove this later) and thus contains only finitely many $\Gamma$-images of $\xi$. Thus the sequence $\gamma_{n}$ contains only finitely many distinct transforms. We see then that for some $\gamma \in \Gamma$, $\gamma \neq I, \gamma(\xi) \in \overline{A_{n}(\xi)}$ for infinitely many $n$ and this contradicts (5) since $\xi \in D_{n}$. 
It remains only to show that

$$
\{z: P(z, w)<P(\xi, w)\} \cap\left(\bigcup_{n=1}^{\infty} \overline{A_{n}(\xi)}\right)
$$

is compact in $B$. To do this we consider a Möbius transform $U$ mapping $B$ to the upper half-space $\left\{z=\left(z_{1}, z_{2}, \ldots, z_{n}\right): z_{n}>0\right\}$ with $U(w)=\infty$. The $U$ image of the set $\{z: P(z, w)<P(\xi, w)\}$ is the exterior of some horosphere at $\infty$, namely a set of the form $\left\{z=\left(z_{1}, z_{2}, \ldots, z_{n}\right): 0<z_{n}<k\right\}$. The $U$ image of the balls $\overline{A_{n}(\xi)}$ are a sequence of balls each containing $U(\xi)$ on the boundary and with centers approaching $\infty$ in a funnel

$$
\left\{z=\left(z_{1}, z_{2}, \ldots, z_{n}\right): z_{1}^{2}+z_{2}^{2}+\cdots+z_{n-1}^{2}<l\right\} .
$$

The $U$ image of the set (6) is easily seen to be compact in the upper half-space and the proof of Theorem 1 is complete.

5. A classification of limit points. In this section we develop a classification of the limit points of a discrete group which will be helpful in the next section when we consider for what limit points $w$ the region $D_{w}$ is a fundamental region. This classification, although given in terms of the function $P$, is essentially geometric in nature and describes how orbits approach a limit point in terms of horospheres at the point.

Suppose $\Gamma$ is a discrete group, $w \in S$ and $z \in B$, and we consider the set of positive numbers $\{P(\gamma(z), w): \gamma \in \Gamma\}$. If this set is unbounded then, from Lemma 1, we observe that the $\Gamma$-orbit of $z$ approaches $w$ in arbitrary small horospheres. In other words, any horospherical region at $w$ contains infinitely many $\Gamma$-images of $z$. This phenomenon is independent of our choice of $z$ and we say in this case that $w$ is a horospherical limit point for $\Gamma$ and write $w \in H$.

Suppose now that $\sup \{P(\gamma(z), w): \gamma \in \Gamma\}$ is finite and is not attained. In this case there is a critical horosphere at $w$ with the property that the horospherical region it determines has no equivalents of $z$ in its closure but that any larger horospherical region meets the orbit of $z$ infinitely often. We say in this case that $w$ is a Garnett point with respect to the orbit of $z$ and write $w \in g_{z}$.

We remark that the notion of horospherical limit point was first introduced by Sullivan, and that of Garnett point by Lucy Garnett - the reader is referred to Sullivan's paper [6] for an account. The existence of Garnett points was first established, for Fuchsian groups, in [3]. At the end of this section we give an example to show that Garnett points exist for discrete groups in all dimensions and that $g_{z}$ varies with $z$.

The only case now left to consider is when $\sup \{P(\gamma(z), w): \gamma \in \Gamma\}$ is finite and attained. We have the following result.

LEMMA 3. If $\Gamma$ is a discrete group, $w \in S, z \in B$ and $z$ is not fixed by any element of $\Gamma$ except the identity, then $P(z, w) \geqslant P(\gamma(z), w)$ for all $\gamma \in \Gamma$ if and only if $w \in \overline{D_{z}}$.

Proof. If $P(z, w) \geqslant P(\gamma(z), w)$ for all $\gamma \in \Gamma$ then we see that the horosphere at $w$ through $z$ determines a horospherical region, say $A$, which does not meet the orbit of 
$z$. Now suppose $\xi$ is on the geodesic ray $\sigma$ which starts at $z$ and ends at $w$. The sphere $\{x: \rho(x, \xi)=\rho(z, \xi)\}$ and the horosphere $\partial A$ both have $\sigma$ as an interior normal at $z$. Thus the sphere is internally tangent to the horosphere and the ball $\{x: \rho(x, \xi)<$ $\rho(z, \xi)\}$ is contained in the horospherical region $A$ and thus contains no $\Gamma$-images of $z$. Thus the point $\xi$ belongs to $D_{z}$ and, since this is true for all $\xi$ on $\sigma$, we see that $w$ $\in \overline{D_{z}}$.

In the opposite direction we may reverse the argument provided we can show that $A=\cup_{\xi \in \sigma}\{x: \rho(x, \xi)<\rho(z, \xi)\}$. We have already seen that the right-hand side is a subset of the left-hand side. Now choose $x \in A$, let $H$ be the horosphere at $w$ through $x$ and $\sigma^{\prime}$ the geodesic segment from $x$ to $w$. The portion of $\sigma$ in $A$ but exterior to $H$ has hyperbolic length $\varepsilon$, say. Now if $\xi$ is chosen on $\sigma$ and $\xi^{\prime}$ on $\sigma^{\prime}$ with $|\xi|=\left|\xi^{\prime}\right|$ then clearly $\rho(z, \xi)=\varepsilon+\rho\left(x, \xi^{\prime}\right)$. We simply choose $\xi$, $\xi^{\prime}$ of modulus so close to 1 that $\rho\left(\xi, \xi^{\prime}\right)<\varepsilon / 2$ and it follows that

$$
\rho(x, \xi)<\rho\left(x, \xi^{\prime}\right)+\rho\left(\xi, \xi^{\prime}\right)<\rho\left(x, \xi^{\prime}\right)+\varepsilon=\rho(z, \xi) .
$$

Thus every point of $A$ belongs to a ball $\{x: \rho(x, \xi)<\rho(z, \xi)\}$ for some $\xi \in \sigma$.

For a nonfixed point $z$ of $B$, the set of points of $S$ which are $\Gamma$-equivalent to boundary points of $D_{z}$ is commonly denoted $E_{z}$. Thus $E_{z}=\cup_{\gamma \in \Gamma} \gamma\left(\overline{D_{z}} \cap S\right)$ and we have the following immediate corollary of the results in this section. This was first observed by Sullivan [6] and is included for completeness.

THEOREM A. If $\Gamma$ is a discrete group and $z \in B$ is not fixed by any element of $\Gamma$ except the identity then $S=H \cup g_{z} \cup E_{z}$.

We conclude this section with an example.

THEOREM 2. In any dimension $n(\geqslant 2)$ we may find a discrete group $\Gamma$ and $z_{1}$, $z_{2} \in B$ such that

(i) $g_{z_{1}} \neq \varnothing$,

(ii) $g_{z_{1}} \neq g_{z_{2}}$.

Proof. For convenience we work in the upper half-space of $\mathbf{R}^{n}$ and mimic the proof for the two-dimensional case given in [3]. For integer $j \geqslant 2$ define the integers

$$
r_{j}=(j !)^{2}, \quad u_{j}=2 \sum_{i=1}^{j-1} r_{i}+r_{j}
$$

and the points of $\mathbf{R}^{n}$

$$
x_{j}=\left(u_{j}, 0,0, \ldots, 0\right), \quad x_{j}^{\prime}=\left(-u_{j}, 0,0, \ldots, 0\right) .
$$

Now define the spheres

$$
S_{j}=\left\{x \in \mathbf{R}^{n}:\left|x-x_{j}\right|=r_{j}\right\}, \quad S_{j}^{\prime}=\left\{x \in \mathbf{R}^{n}:\left|x-x_{j}^{\prime}\right|=r_{j}\right\} .
$$

Denote by $\gamma_{j}$ the Möbius transformation of $\mathbf{R}^{n}$ which comprises an inversion in $S_{j}$ followed by the reflection $\left(x_{1}, x_{2}, \ldots, x_{n}\right) \rightarrow\left(-x_{1}, x_{2}, \ldots, x_{n}\right)$. For each $j, \gamma_{j}$ preserves the upper half-space and the isometric spheres of $\gamma_{j}, \gamma_{j}^{-1}$ are $S_{j}, S_{j}^{\prime}$, respectively. 
Observe that the collection of spheres defined above is pairwise exterior (except that $S_{j}, S_{j+1}$ are externally tangent) and so the group $\Gamma$ generated by the $\gamma_{j}, j \geqslant 2$, is discrete and the Ford region for its action in the upper half-space is precisely that part of upper half-space which is exterior to all the $S_{j}, S_{j}^{\prime}, j \geqslant 2$. If $\lambda=(0,0,0, \ldots, 0,1)$ in $\mathbf{R}^{n}$ and $f$ is the function on $\mathbf{R}^{n}$ which assigns to each point its last coordinate, then we note that $f(\gamma(\lambda))<1$ if $\gamma \in \Gamma, \gamma \neq I$ because $\lambda$ is exterior to all isometric spheres in $\Gamma$. An easy calculation shows that

$$
f\left(\gamma_{j}(\lambda)\right)=r_{j}^{2} /\left|\lambda-x_{j}\right|^{2}=r_{j}^{2} / u_{j}^{2}+1>(1+2 / j)^{-2}
$$

for $j \geqslant 2$.

If $\gamma \in \Gamma$ we may write $\gamma$ uniquely as a word in the generators $\gamma_{2}, \gamma_{3}, \ldots$ and let $\exp \gamma$ be the sum of the exponents appearing in this word. Define a subgroup $G$ of $\Gamma$ by $G=\{\gamma \in \Gamma$ : $\exp \gamma=0\}$. The closed horospherical region at $\infty$ given by $\{x$ : $f(x) \geqslant 1\}$ meets the $\Gamma$-orbit of $\lambda$ in precisely the point $\lambda$. Thus, setting $w=\gamma_{2}(\lambda)$ we see that $\{x: f(x) \geqslant 1\} \cap G(w)=\varnothing$. However, $f\left(\gamma_{j}(\lambda)\right) \rightarrow 1$ as $j \rightarrow \infty$ and, since we may write $\gamma_{j}(\lambda)=\gamma_{j} \gamma_{2}^{-1}(w)(j \geqslant 3)$, we see that $\gamma_{j}(\lambda)$ are in the $G$-orbit of $w$. Thus $\infty$ is a Garnett point for $G$ with respect to the orbit of $w$ and, clearly, it is not a Garnett point with respect to the orbit of $\lambda$. This proves the theorem.

6. A class of fundamental regions. In this section we will classify those limit points $w$ for which $D_{w}$ is a fundamental region. We first remark that, although the hyperbolic metric in $B$ does not extend to the boundary, the topology does-via the well-known concept of horospherical neighborhoods of boundary points. Thus, in a topological sense, $D_{w}$ can be regarded quite naturally as a 'Dirichlet region' even when $w \in S$. In this section we prove

TheOREm 3. Let $\Gamma$ be a discrete group and $w \in B \cup S$. Then the interior of $D_{w}$ is a fundamental region for $\Gamma$ if and only if

(i) $w$ is not fixed by any element of $\Gamma$ (except the identity),

(ii) $w$ is not a horospherical limit point and

(iii) $w$ is not a Garnett point with respect to any orbit.

Proof. Suppose $\gamma$ fixes $w(\gamma \neq I)$ and $z \in D_{w}$. We observe that $P(\gamma(z), w)=$ $P(\gamma(z), \gamma(w))$ and so, from (1), (2),

$$
P(\gamma(z), w)=P(z, w)\left|\gamma^{\prime}(w)\right|^{-1} .
$$

Since $z \in D_{w}, P(z, w)>P(\gamma(z), w)$ and thus $\left|\gamma^{\prime}(w)\right|>1$. Similarly, since $\gamma^{-1}$ also fixes $w$, we have $\left|\left(\gamma^{-1}\right)^{\prime}(w)\right|>1$ and $w$ is exterior to the isometric spheres of both $\gamma$ and $\gamma^{-1}$. This clearly contradicts the fact that $\gamma$ fixes $w$ and we deduce that if (i) fails then $D_{w}=\varnothing$.

If condition (ii) fails it follows immediately from the definition that $D_{w}=\varnothing$.

If condition (iii) fails then for some $z \in B, w \in g_{z}$ and so $\sup \{P(\gamma(z), w): \gamma \in \Gamma\}$ is not attained. This means that the $\Gamma$-orbit of $z$ does not meet $\bar{D}_{w}$ and consequently does not meet $\overline{\text { Int } D_{w}}$. Thus in this case the interior of $D_{w}$ is not a fundamental region for $\Gamma$. 
We recall that $D_{w}$ cannot contain two $\Gamma$-equivalent points; thus, the same is true of its interior. To complete the proof of the theorem we must therefore show that conditions (i)-(iii) together imply that any point of $B$ has a $\Gamma$-equivalent in the closure of the interior of $D_{w}$. Given any $z \in B$ these conditions imply that for some $\gamma_{1} \in \Gamma$

$$
P\left(\gamma_{1}(z), w\right) \geqslant P(\gamma(z), w) \quad \text { all } \gamma \in \Gamma .
$$

We may as well assume that $\gamma_{1}$ is the identity and thus we need to show that if

$$
P(z, w) \geqslant P(\gamma(z), w) \text { all } \gamma \in \Gamma,
$$

then $z$ is in the closure of the interior of $D_{w}$.

For any $\gamma \in \Gamma$ the sphere $L(\gamma, w)=\{x \in B: P(x, w)=P(\gamma(x), w)\}$ divides $B$ into two convex subspaces

and

$$
A(\gamma, w)=\{x \in B: P(x, w)>P(\gamma(x), w)\}
$$

$$
C(\gamma, w)=\{x \in B: P(x, w)<P(\gamma(x), w)\} .
$$

It is easy to check that $w \in A(\gamma, w)$ if $w \in B$ and that $w$ is on the boundary of $A(\gamma, w)$ if $w \in S$. It follows immediately from this and convexity that if $z$ satisfies (7) then all points of the open geodesic ray connecting $z$ to $w$ belong to $A(\gamma, w)$ for all $\gamma$ in $\Gamma$. Thus, since $D_{w^{\prime}}=\bigcap_{\gamma \in \Gamma} A(\gamma, w)$, the open geodesic ray joining $z$ to $w$ is contained in $D_{w}$.

Now given $z$ satisfying (7) we find points $z_{i}, 1 \leqslant i \leqslant n-1$, of $B$ each of which satisfies (7) and with the additional property that the interior of the noneuclidean convex hull of the points $z, w, z_{1}, \ldots, z_{n-1}$ is an open set in $\mathbf{R}^{n}$. It is clear that this can be done since every point of $B$ has an equivalent satisfying (7). By our remarks above, all points of the interior of this convex hull lie in $D_{w}$ and so $z$ is in the closure of the interior of $D_{k}$. This completes the proof of the theorem.

We conclude this section by considering the region $D_{\infty}$ for each of the groups $\Gamma$ and $G$ defined in the proof of Theorem 2. If we denote by $D$ the region of the upper half-space exterior to all the spheres $S_{j}, S_{j}^{\prime}, j=2,3, \ldots$, then it is rather easy to show that, for the group $\Gamma, D_{\infty}=D$ which is an open, convex, locally-finite fundamental region. Since $\infty$ is a limit point for $\Gamma$ we see that our construction does indeed yield new examples of fundamental regions based at limit points.

To find the region $D_{\infty}$ for the group $G$ of Theorem 2 we note first that $D_{\infty} \supset D$ (since $G$ is a subgroup of $\Gamma$ ). Calculations as performed in the proof of Theorem 2 show that for any $x$ in the upper half-space $f\left(\gamma_{j}(x)\right) \rightarrow f(x), f\left(\gamma_{j}^{-1}(x)\right) \rightarrow f(x)$ as $j \rightarrow \infty$. Now if $x$ is interior to $S_{i}$, say, then $f\left(\gamma_{i}(x)\right)>f(x)$ and so, for $j$ large enough, $f\left(\gamma_{j}^{-1} \gamma_{i}(x)\right)>f(x)$ and, since $\gamma_{j}^{-1} \gamma_{i} \in G$, we see that $x$ is outside the closure of $D_{x}$. Thus $\bar{D} \supset D_{\infty} \supset D$. If $x \in S_{i}$ then $f(\gamma(x))<f(x)$ for all $\gamma$ in $\Gamma$ except $I$ and $\gamma_{i}$, but $\gamma_{i} \notin G$ and so $x \in D_{\infty}$. Thus we have shown that, for the group $G, D_{\infty}=\bar{D}$.

7. The set of Garnett points. Given a discrete group $\Gamma$ acting in $B$ we define $G=\cup_{z \in B} g_{z}$.

In [6] Sullivan shows explicitly that $g_{z}$ has zero $(n-1)$-dimensional Lebesgue measure as a subset of $S$. The much stronger result that $G$ has zero measure is also 
implicit in Sullivan's paper but requires results on the ergodic action of the group on the sphere $S$. In this section we give a more direct and elementary proof of this result. It should be noted that, in dimension two, the result is implicit in the work of Pommerenke [4] but that his approach requires a result of Frostman on Blaschke products and will not generalize to higher dimensions.

TheOREM B. For any discrete group $\Gamma$ the set $G$ has zero $(n-1)$-dimensional Lebesgue measure as a subset of $S$.

Proof. If $w \in S, z \in B$ and $0<k<1$, then $z$ is interior to the horosphere at $w$ of radius $k$ if and only if $P(z, w)>(1-k) / k$ (see Lemma 1). From the fact that $|z-w|^{2}=(z-w) \cdot(z-w)$ we note that

$$
2 w \cdot z=1+|z|^{2}-|z-w|^{2} \text {. }
$$

From this we have that

$$
|w-z /| z||^{2}=(w-z /|z|) \cdot(w-z /|z|)=2+\left(|z-w|^{2}-|z|^{2}-1\right) /|z| .
$$

Now $P(z, w)>(1-k) / k$ if and only if $|z-w|^{2}<k\left(1-|z|^{2}\right) /(1-k)$. Thus, from the equation above, we deduce that $P(z, w)>(1-k) / k$ if and only if

$$
|w-z /| z||^{2}<(1-|z|)(2 k-1+|z|)|z|^{-1}(1-k)^{-1} \text {. }
$$

Now suppose $\Gamma$ is a discrete group, write $\Gamma=\left\{\gamma_{n}: n=1,2, \ldots\right\}$ and define for $0<k<1$,

$$
\begin{aligned}
I(m, k)=\{\xi \in S: \mid & \xi-\gamma_{m}(0) /\left.\left|\gamma_{m}(0)\right|\right|^{2} \\
& \left.<\left(1-\left|\gamma_{m}(0)\right|\right)\left(2 k-1+\left|\gamma_{m}(0)\right|\right)\left|\gamma_{m}(0)\right|^{-1}(1-k)^{-1}\right\}
\end{aligned}
$$

which is a "disc" on $S$ centered at the radial projection of $\gamma_{m}(0)$.

Thus we have shown that $w \in I(m, k)$ if and only if $\gamma_{m}(0)$ is interior to the horosphere at $w$ of radius $k$. The next lemma provides the measure of the set $I(m, k)$.

Lemma 4. For $\eta \in S$ and $\lambda>0$, set $A=\{x \in S:|x-\eta|<\lambda\}$. Then

$$
w(A)=M \int_{0}^{\mu}(\operatorname{Sin} \theta)^{n-2} d \theta
$$

where $w$ denotes the $(n-1)$-dimensional Lebesgue measure on $S$,

$$
\mu=\operatorname{arc} \operatorname{Cos}\left(1-\lambda^{2} / 2\right)
$$

and $M$ is a constant.

Proof. We introduce polar coordinates in $\mathbf{R}^{n}$ as follows. Given a point $\left(x_{1}, x_{2}, \ldots, x_{n}\right)$ in $\mathbf{R}^{n}$ set $r=\left(x_{1}^{2}+x_{2}^{2}+\cdots+x_{n}^{2}\right)^{1 / 2}$ and, for $j$ satisfying $1 \leqslant j \leqslant n$, define $\theta_{j}$ to be the angle between the $j$ th coordinate vector and

$$
\left(0,0,0, \ldots, 0, x_{j}, x_{j+1}, \ldots, x_{n}\right) \text {. }
$$


Thus

$$
\theta_{j}=\operatorname{arcCos}\left[x_{j} /\left(x_{j}^{2}+\cdots x_{n}^{2}\right)^{1 / 2}\right], \quad \theta_{j} \in[0, \pi] .
$$

Proceeding inductively we can show that, for $1<j \leqslant n$,

$$
x_{j}=r \operatorname{Sin} \theta_{1} \operatorname{Sin} \theta_{2} \cdots \operatorname{Sin} \theta_{j-1} \operatorname{Cos} \theta_{j}
$$

and $x_{1}=r \operatorname{Cos} \theta_{1}$. Note that $\theta_{n}=0$ or $\pi$.

The Jacobian of the transform $\left(r, \theta_{1}, \theta_{2}, \ldots, \theta_{n-1}\right) \rightarrow\left(x_{1}, x_{2}, \ldots, x_{n}\right)$ is calculated inductively to be

$$
r^{n-1}\left(\operatorname{Sin} \theta_{1}\right)^{n-2}\left(\operatorname{Sin} \theta_{2}\right)^{n-3} \cdots\left(\operatorname{Sin} \theta_{n-2}\right) .
$$

Thus the volume element in polar coordinates is

$$
r^{n-1}\left(\operatorname{Sin} \theta_{1}\right)^{n-2}\left(\operatorname{Sin} \theta_{2}\right)^{n-3} \cdots\left(\operatorname{Sin} \theta_{n-2}\right) d \theta_{1} d \theta_{2} \cdots d \theta_{n-1} d r
$$

and the surface area element on the unit sphere is

$$
\left(\operatorname{Sin} \theta_{1}\right)^{n-2}\left(\operatorname{Sin} \theta_{2}\right)^{n-3} \cdots\left(\operatorname{Sin} \theta_{n-2}\right) d \theta_{1} d \theta_{2} \cdots d \theta_{n-1} .
$$

Returning to the proof of the lemma, we may as well assume that $\eta=(1,0, \ldots, 0)$ and then, for $X \in s,|\eta-x|^{2}=2\left(1-x_{1}\right)$ where $x=\left(x_{1}, x_{2}, \ldots, x_{n}\right)$. From this it follows that $A=\left\{x \in S: 1-\lambda^{2} / 2 \leqslant x_{1} \leqslant 1\right\}$. Recall however, that $x_{1}=\operatorname{Cos} \theta_{1}$ and the lemma follows when we integrate the surface area element over $A$ and set

$$
M=\int_{0}^{\pi} \int_{0}^{\pi} \cdots \int_{0}^{\pi}\left(\operatorname{Sin} \theta_{2}\right)^{n-3}\left(\operatorname{Sin} \theta_{3}\right)^{n-4} \cdots\left(\operatorname{Sin} \theta_{n-2}\right) d \theta_{2} \cdots d \theta_{n-1}
$$

which is an absolute constant-namely, half the $(n-2)$-dimensional Lebesgue measure of the unit sphere in $\mathbf{R}^{n-1}$.

As $m$ increases to infinity, the "disc" $I(m, k)$ shrinks to a point and, from Lemma 4 , we see that

$$
w(I(m, k)) \sim \frac{M}{n-1}\left\{2 k\left(1-\left|\gamma_{m}(0)\right|\right)(1-k)^{-1}\right\}^{(n-1) / 2}
$$

as $m \rightarrow \infty$. To see this, replace $\operatorname{Sin} \theta$ by $\theta$ in the integral of Lemma 4 , note that $\mu \sim \lambda$ and that $\lambda$ is the square root of

$$
\left(1-\left|\gamma_{m}(0)\right|\right)\left(2 k-1+\left|\gamma_{m}(0)\right|\right)\left|\gamma_{m}(0)\right|^{-1}(1-k)^{-1} \text {. }
$$

Now select two numbers $0<k_{1}<k_{2}<1$ and note that $I\left(m, k_{1}\right) \subset I\left(m, k_{2}\right)$. From the above, there exists $\delta>0$ such that, for $m$ large enough,

$$
w\left(I\left(m, k_{1}\right)\right) / w\left(I\left(m, k_{2}\right)\right)>\delta .
$$

We claim that the set of points lying in infinitely many $I\left(m, k_{1}\right)$ has the same $w$-measure as the set lying in infinitely many $I\left(m, k_{2}\right)$. We follow Sprindzuk [5, p. 21] and define

$$
J=\bigcap_{l=1}^{\infty} \bigcup_{m=l}^{\infty} I\left(m, k_{2}\right) ; \quad B_{l}=\bigcup_{m=l}^{\infty} I\left(m, k_{1}\right) ; \quad D_{l}=J \backslash B_{l}
$$


and it suffices to show that every $D_{l}$ is of measure zero. If this is not the case then $D_{l}$ contains a point $x_{0}$ of metric density. Since $x_{0} \in J$ for infinitely many $m$ we have $x_{0} \in I\left(m, k_{2}\right)$ and for such $m$

$$
w\left[D_{m} \cap I\left(m, k_{2}\right)\right] \sim w\left[I\left(m, k_{2}\right)\right] \quad(m \rightarrow \infty)
$$

since $w\left[I\left(m, k_{2}\right)\right] \rightarrow 0$ as $m \rightarrow \infty$. On the other hand, the sets $D_{m}$ and $I\left(m, k_{1}\right)$ do not intersect if $m \geqslant l$, and hence $D_{m} \cap I\left(m, k_{2}\right)$ and $I\left(m, k_{1}\right)$ are nonintersecting subsets of $I\left(m, k_{2}\right)$. Therefore

$$
\begin{aligned}
w\left[I\left(m, k_{2}\right)\right] & \geqslant w\left[I\left(m, k_{1}\right)\right]+w\left[D_{m} \cap I\left(m, k_{2}\right)\right] \\
& \geqslant \delta w\left[I\left(m, k_{2}\right)\right]+w\left[D_{m} \cap I\left(m, k_{2}\right)\right]
\end{aligned}
$$

and so $w\left[D_{m} \cap I\left(m, k_{2}\right)\right] \leqslant(1-\delta) w I\left(m, k_{2}\right)$ which contradicts (8).

We have shown that for $k_{1}, k_{2}$ satisfying $0<k_{1}<k_{2}<1$ the sets $\lim \sup I\left(m, k_{1}\right)$, $\lim \sup I\left(m, k_{2}\right)$ have the same $w$ measure on $S$. The horospherical limit set $H$ is given by

$$
H=\bigcap_{p=2}^{\infty} \lim \sup I(m, 1 / p)
$$

which, therefore, has the same measure as the set

$$
A=\bigcup_{p=2}^{\infty} \lim \sup I(m, 1-1 / p) .
$$

However, $A$ comprises those points of $S$ at which some horosphere contains infinitely many images of the origin in its interior. Thus $G \subset A \backslash H$ and we have $w(G)=0$. This completes the proof of Theorem B.

\section{REFERENCES}

1. L. V. Ahlfors, Hyperbolic motions, Nagoya Math. J. 29 (1967), 163-166.

2. A. F. Beardon and P. J. Nicholls, Ford and Dirichlet regions for Fuchsian groups, Canad. J. Math. 34 (1982), 806-815.

3. P. J. Nicholls, Garnett points for Fuchsian Groups, Bull. London Math. Soc. 12 (1980), 216-218.

4. Ch. Pommerenke, On the Green's function of Fuchsian groups, Ann. Acad. Sci. Fenn. A I Math. 2 (1976), 409-427.

5. V. G. Sprindzuk, Metric theory of Diophantine approximation, Wiley, New York, 1979.

6. D. Sullivan, On the ergodic theory at infinity of an arbitrary discrete group of hyperbolic motions, in Riemann Surfaces and Related Topics, Ann. of Math. Studies, no. 97, Princeton Univ. Press, Princeton, N. J., 1980, pp. 465-496.

Department of Mathematics, Northern Illinois University, DeKalb, Illinois 60115 